\title{
BMJ
}

\section{Thrombolysis in very elderly people: controlled comparison of SITS International Stroke Thrombolysis Registry and Virtual International Stroke Trials Archive}

\author{
Nishant K Mishra, doctoral candidate, ${ }^{1}$ Niaz Ahmed, neurologist, ${ }^{2}$ Grethe Andersen, neurologist, ${ }^{3}$ José A Egido, \\ neurologist, ${ }^{4}$ Perttu J Lindsberg, professor of applied neurology, ${ }^{5}$ Peter A Ringleb, neurologist, ${ }^{6}$ Nils G \\ Wahlgren, professor of neurology, ${ }^{2}$ Kennedy R Lees, professor of cerebrovascular medicine ${ }^{1}$ for the VISTA \\ and SITS collaborators
}

${ }^{1}$ Acute Stroke Unit, University Department of Medicine and Therapeutics, Gardiner Institute, Western Infirmary and Faculty of Medicine, University of Glasgow, Glasgow G11 6NT, UK

${ }^{2}$ Department of Neurology, Karolinska University Hospital,

Karolinska Institutet 17176 Stockholm, Sweden

${ }^{3}$ Department of Neurology, Aarhus University Hospital, Aarhus, Denmark

${ }^{4}$ Department of Neurology, Hospital San Carlos, Spain ${ }^{5}$ Department of Neurology, Helsinki University Central Hospital, and Program of Molecular Neurology, University of Helsinki, Helsinki, Finland

${ }^{6}$ Department of Neurology, University Hospital of Heidelberg, Heidelberg, Germany

Correspondence to: K R Lees k.r.lees@clinmed.gla.ac.uk

Cite this as: BMJ 2010;341:c6046 doi:10.1136/bmi.c6046

\section{ABSTRACT}

Objective To assess effect of age on response to alteplase in acute ischaemic stroke.

Design Adjusted controlled comparison of outcomes between non-randomised patients who did or did not undergo thrombolysis. Analysis used Cochran-MantelHaenszel test and proportional odds logistic regression analysis.

Setting Collaboration between International Stroke Thrombolysis Registry (SITS-ISTR) and Virtual International Stroke Trials Archive (VISTA).

Participants 23334 patients from SITS-ISTR (December 2002 to November 2009) who underwent thrombolysis and 6166 from VISTA neuroprotection trials (1998-2007) who did not undergo thrombolysis (as controls). Of the 29500 patients (3472 aged $>80$ ("elderly," mean 84.6), data on 272 patients were missing for baseline National Institutes of Health stroke severity score, leaving 29228 patients for analysis adjusted for age and baseline severity.

Main outcome measures Functional outcomes at 90 days measured by score on modified Rankin scale.

Results Median severity at baseline was the same for patients who underwent thrombolysis and controls (median baseline stroke scale score: 12 for each group, $P=0.14 ; n=29228$ ). The distribution of scores on the modified Rankin scale was better among all thrombolysis patients than controls (odds ratio 1.6, 95\% confidence interval 1.5 to 1.7; Cochran-Mantel-Haenszel P<0.001). The association occurred independently among patients aged $\leq 80$ (1.6, 1.5 to 1.7; $P<0.001 ; n=25789)$ and in those aged $>80(1.4,1.3$ to 1.6 ; $P<0.001 ; n=3439)$. Odds ratios were consistent across all 10 year age ranges above 30 , and benefit was significant from age 41 to 90 ;

dichotomised outcomes (score on modified Rankin scale $0-1 v 2-6$; 0-2 $v$ 3-6; and 6 (death) $v$ rest) were consistent with the results of the ordinal analysis.

Conclusions Outcome in patients with acute ischaemic stroke is significantly better in those who undergo thrombolysis compared with those who do not. Increasing age is associated with poorer outcome but the association between thrombolysis treatment and improved outcome is maintained in very elderly people. Age alone should not be a barrier to treatment.

\section{INTRODUCTION}

Thrombolysis for acute ischaemic stroke has proved benefits, ${ }^{1-5}$ but data from randomised trials in patients aged over 80 are limited. About $30 \%$ of acute stroke occurs in people aged over $80 .^{6-8}$ The NINDS (National Institute of Neurological Disorders and Stroke) trial initially restricted enrolment to patients aged up to $80 . .^{9}$ The age criterion was lifted after they enrolled 188 patients in part A of the trial, but they enrolled only 42 very elderly patients. ${ }^{2}$ All ECASS (European Cooperative Acute Stroke Study) trials applied an upper age limit of $80,{ }^{310}$ and recent studies with desmoteplase (a fibrin specific plasminogen activator $)^{1112}$ also excluded elderly patients. Thus, up to now, the European Medicines Evaluation Agency has not approved thrombolysis with alteplase among patients aged above $80 .{ }^{4}$ Many experienced centres treat elderly patients but others observe the terms of product approval. ${ }^{13-16}$

The main reasons for withholding treatment from very elderly patients in clinical practice are fears that advancing age is associated with poorer prognosis with greater risk for haemorrhage and in hospital mortality. ${ }^{17-19}$ Conversely, a meta-analysis of pooled thrombolysis data concluded that the risks of symptomatic intracerebral haemorrhage did not increase among elderly patients, despite less favourable outcomes. ${ }^{1520}$ Less favourable outcomes are expected to occur in elderly patients, mostly because of comorbidity. ${ }^{1921}$

The proportion of older people is rising in our society, ${ }^{6-8}$ and the proportion of those undergoing thrombolysis will decline in the future if patients aged over 80 are not treated. ${ }^{22}$ In the United Kingdom alone, 
the population aged over 80 has doubled since $1982,{ }^{23}$ and life expectancy has risen in the rest of Europe and in other countries. ${ }^{24}$ Effective treatments, however, should not be withheld from older people in the absence of compelling data suggesting unacceptable risk or proved lack of benefit. We hypothesised that clinical practice over the past decade would have been sufficiently diverse to allow analysis of existing rigorously collected clinical data ${ }^{2526}$ to construct a comparison of thrombolysis against matched controls, with the possibility of adjusting for any imbalance in severity. We anticipated that use in older people would be sufficiently common to assess the influence of age on any association of stroke outcome with thrombolysis.

\section{METHODS}

\section{Data source and patients}

We collated the data of stroke patients who underwent thrombolysis through the SITS-ISTR (Safe Implementation of Treatment in Stroke-International Stroke Thrombolysis Registry, www.sitsinternational.org, held at Karolinska University Hospital, Stockholm) from December 2002 to November 2009. Our control group comprised untreated stroke patients from neuroprotection trials conducted from 1998 to 2007 and held within the Virtual International Stroke Trials Archive, VISTA (www.vista.gla.ac.uk). ${ }^{26}$

The SITS-ISTR is an ongoing internet based, academic driven, interactive thrombolysis register. The methods of the register, including the procedure for data collection and management, identification of patients, and verification of source data, has been described previously. ${ }^{27528}$ In brief, it is a prospective open multinational observational monitoring registry for clinical centres using thrombolysis and other interventions for the treatment of acute ischaemic stroke. The registry is open to all countries, and collects data on patients who receive thrombolytic therapy for acute ischaemic stroke. ${ }^{528}$

VISTA is a collaborative, not-for-profit, register of stroke trials. ${ }^{26}$ The treatments studied in these trials range from putative neuroprotectants through anticoagulants and thrombolytic agents to simple rehabilitation measures. ${ }^{26}$ Unfortunately, the effects of the neuroprotectants have largely been indistinguishable from placebo. ${ }^{29}$ All trials in VISTA hold necessary review board and regulatory approvals, and all patients agreed to participation. The archive holds only anonymised data, and the source trial is not disclosed as per VISTA guidelines. ${ }^{26}$ For this analysis, we sought data from the archive from a group of trials in which the investigated drug was a putative neuroprotectant that was neither vasoactive nor interfered with clotting or from placebo groups. Hence, the "controls" were patients who did not receive alteplase in neuroprotection trials but received either placebo or a neuroprotective drug as per the randomisation protocol for each contributing trial. From both of these sources, we collated the demographics, clinical data, and information of functional outcome as measured by the score on the modified Rankin scale after 90 days.

\section{Patient sample}

We downloaded data on 28136 patients registered in SITS-ISTR from 25 December 2002 to 2 November 2009 and extracted information on 23336 who had completed 90 day follow-up. Two patients were excluded because of inexact information on age, leaving 23334. Participating centres agreed to record details of every patient treated with intravenous alteplase at that centre and to enter baseline data as soon as possible after treatment, adding outcomes as they occurred. $^{27}$

We collated data on 9665 patients from VISTA. Of these, 6371 patients who had ischaemic stroke and did not undergo thrombolysis were selected as controls. We excluded 205 patients with no information on functional outcomes at 90 days, leaving 6166 for analysis.

Of the 29500 patients $(n=3472$ aged $>80)$, data on baseline National Institutes of Health stroke scale score were missing for 272 patients, leaving 29228 for analysis adjusted for age and baseline severity (fig 1 ).

\section{Statistical analysis}

We compared outcome at 90 days in patients who received intravenous thrombolysis and controls for the whole cohort. We repeated the comparison among patients aged $\leq 80$ and $>80$ years. We then examined the association of thrombolysis treatment with outcome within various age groups $(<21,21-30$, 31-40, 41-50, 51-60, 61-70, 71-80, 81-90, and 91-100) to illustrate the strength of evidence across the full age range.

For each comparison, we looked at the overall distribution of all seven categories of scores on the modified Rankin scale in the two groups at day 90. The modified Rankin scale is an ordinal score used as a measure of functional outcomes in patients with stroke (see box). ${ }^{3031}$

\section{Scores on modified Rankin scale}

\section{$0=$ no symptoms from stroke}

$1=$ no severe disability, despite symptoms

$2=$ slight disability in which patients are unable to do all previous activities but able to look after themselves without help

$3=$ moderate disability that requires some help, but patients can walk by themselves

4=moderately severe disability in which patients are unable to walk without assistance and need help for bodily needs

5=bedbound patients who are incontinent or require personal attention

$6=$ death 
To test for a significant association of distribution of outcome with exposure to thrombolysis we used the Cochran-Mantel-Haenszel statistic, adjusting for age and baseline score on the National Institutes of Health stroke scale as continuous variables. ${ }^{32}{ }^{33}$ We had two reasons for our choice of baseline factors for adjustment. Firstly, age and baseline severity measured by National Institutes of Health stroke scale are the two most powerful prognostic factors for stroke outcome and are usually included in analyses of outcome distribution. ${ }^{32-36}$ Secondly, we had data on age and the stroke scale for our entire sample, whereas data on other factors of potential interest were incomplete. We also undertook a sensitivity analysis by considering the combined effect of the variables that differed significantly at baseline.

Our objective was mainly to undertake an ordinal distribution or "shift" analysis, which is an efficient endpoint analysis technique accepted by the European Agency for the Evaluation of Medicinal Products (EMEA).$^{37}$ Shift analysis is considered to be better than dichotomisation of endpoint measures, though there are differences of opinion. ${ }^{38-43}$ Dichotomisation is criticised for the statistical information it discards, whereas shift analysis is especially useful when the treatment effect is mild or uniform, or both, across all Rankin categories, though in larger datasets it can sometimes incorrectly seem to violate proportionality assumptions. ${ }^{38-4044}$ This is because the test for proportionality assumptions is described as "sensitive to sample size, such that large samples may produce statistically significant $\mathrm{P}$ values when in fact there is little practical difference between the cut-point-specific estimates." ${ }^{45}$ Hence, though we planned to undertake examination of outcomes by proportional odds logistic regression analysis we also elected to undertake secondary analyses, dichotomising the Rankin scores 0-1 $v$ rest, $0-2 v$ rest, and dead $v$ survivors, to allow

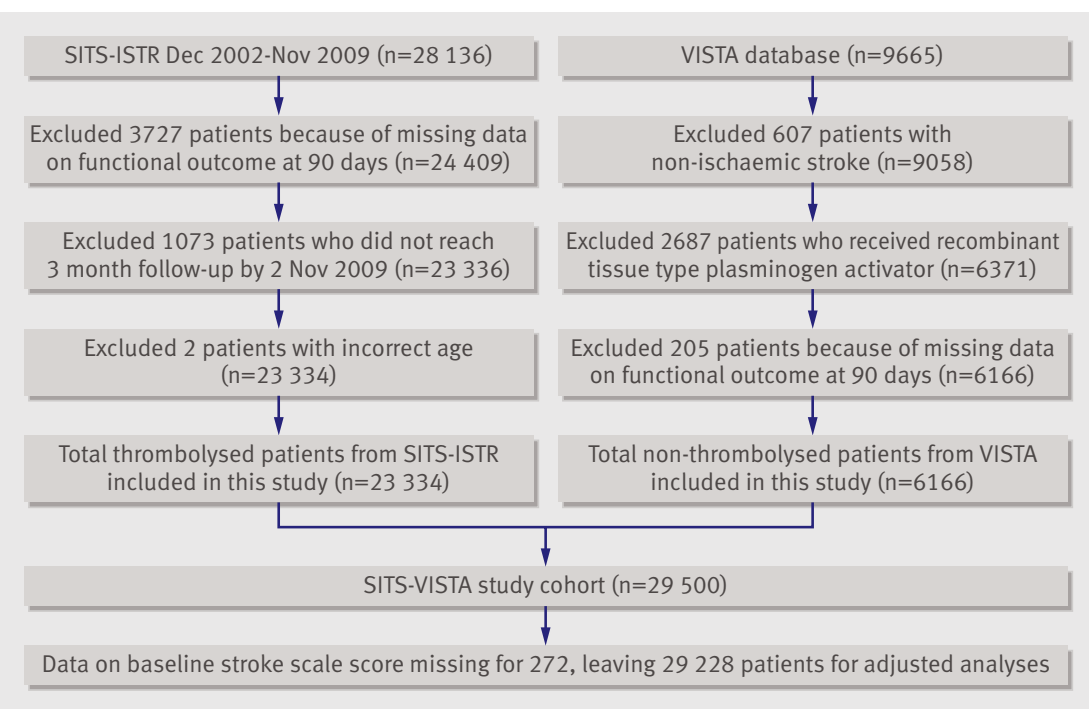

Fig 1 | Description of analysed patients from two data sources comparability of our findings with the other published data. ${ }^{46}$ Odds ratios in our analysis express the common odds of an improved distribution of outcome in association with treatment with alteplase.

The Cochran-Mantel-Haenszel test and proportional odds logistic regression analysis were undertaken with SAS 9.2 software and other analyses by StatsDirect software. We describe our findings in accordance with the STROBE guidelines. ${ }^{47}$

Reliable information on symptomatic intracerebral haemorrhage was not available from VISTA controls as post-treatment imaging was not routinely carried out in neuroprotection trials in patients who had not been treated with alteplase. However, we compared the rates of symptomatic intracerebral haemorrhage for the definitions used within SITS (local or remote parenchymal haemorrhage type 2 on the imaging scan 22-36 hours after treatment, combined with a neurological deterioration of 4 or more points on the National Institutes of Health stroke severity scale from baseline, or from the lowest National Institutes of Health stroke severity score between baseline and 24 hours, or leading to death) and the NINDS study (any intracranial haemorrhage in the post-thrombolysis imaging scans if it was not seen on a previous imaging scan and any decline in neurological status) for younger and older patients of the SITS-ISTR registry.

\section{RESULTS}

All stroke patients were treated as per institutional practice and stroke guidelines acceptable at the point of their treatment. The table shows the baseline characteristics. Baseline severity of stroke was similar between patients who did and did not undergo thrombolysis among those aged $>80(\mathrm{P}=0.6)$ and $\leq 80(\mathrm{P}=0.3)$.

Independently, baseline National Institutes of Health stroke scale accounted for $25.5 \%$ and age for $7.4 \%$ of the variation in 90 day outcome by modified Rankin scale (both $\mathrm{P}<0.001)$ and were included in all models, together explaining $29.6 \%$ of the variation.

\section{Overall outcome}

Across our whole sample, the distribution of scores on the modified Rankin scale at three months was betterthat is, more patients had lower scores-among those who underwent thrombolysis (fig 2). The overall odds ratio was $1.6(95 \%$ confidence interval 1.5 to 1.7 ; $\mathrm{P}<0.001$ ) (fig 3).

\section{Outcomes among patients aged $\leq 80$}

Treatment with thrombolysis was associated with a significantly more favourable distribution of scores on the modified Rankin scale at three months (fig 2). The adjusted odds ratio was 1.6 (1.5 to 1.7$) ; \mathrm{P}<0.001$; $\mathrm{n}=$ 25789 (fig 3). P for test of proportionality assumption was $<0.05$. The unadjusted odds ratio was 1.5 (1.4 to 1.6) $(\mathrm{n}=26$ 028). Dichotomised outcomes were also significantly more favourable for thrombolysed patients than controls (1.9 (1.7 to 2.0) for score $0-2 v 3-6$ on 


\section{Baseline characteristics of patients in SITS*-VISTA}

$$
\text { Thrombolysis }
$$

Control

$P$ value

Mean (SD) and median (range) age (years)

\begin{tabular}{lccc}
\hline All & $67.1(12.4), 69(10-98) ; n=23334$ & $70.1(12.2), 72(21-101) ; n=6166$ & $<0.001$ \\
\hline$\leq 80$ & $65.3(11.63), 68(10-80) ; n=21099$ & $66.5(10.7), 69(21-80) ; n=4929$ & $<0.001$ \\
\hline 180 & $84.4(3.25), 84(81-98) ; n=2235$ & $84.84(3.36), 84(81-101) ; n=1237$ & $<0.001$ \\
\hline
\end{tabular}

\section{No (\%) of men}

\begin{tabular}{lccc}
\hline All & $13594 / 23334(58.3)$ & $3271 / 6166(53.0)$ & $<0.001$ \\
\hline$\leq 80$ & $12744 / 21099(60.4)$ & $2783 / 4929(56.5)$ & $<0.001$ \\
\hline$>80$ & $850 / 2235(38.0$ & $488 / 1237(39.5)$ & 0.41
\end{tabular}

Median (range) baseline score on National Institutes of Health stroke scale

\begin{tabular}{lcll}
\hline All & $12(0-42), n=23062$ & $12(2-37), n=6166$ & 0.14 \\
\hline$\leq 80$ & $12(0-42), n=20860$ & $11(2-32), n=4929$ & 0.32 \\
\hline 180 & $14(0-39), n=2202$ & $14(2-37), n=1237$ & 0.61
\end{tabular}

No (\%) who had previously taken antithrombotics

\begin{tabular}{llcc}
\hline All & $8776 / 22792(38.5)$ & $1267 / 2968(42.7)$ & $<0.001$ \\
\hline$\leq 80$ & $7537 / 20623(36.5)$ & $977 / 2414(40.5)$ & $<0.001$ \\
\hline$>80$ & $1239 / 2169(57.1 \%$ & $290 / 554(52.3)$ & 0.04
\end{tabular}

No (\%) with known diabetes mellitus

\begin{tabular}{lccc}
\hline All & $3962 / 22968(17.2)$ & $1449 / 5896(24.6)$ & $<0.001$ \\
\hline$\leq 80$ & $3570 / 20784(17.2)$ & $1203 / 4704(25.6)$ & $<0.001$ \\
\hline$>80$ & $392 / 2184(17.9)$ & $246 / 1192(20.6)$ & 0.06 \\
\hline
\end{tabular}

No (\%) with previous stroke

\begin{tabular}{lccc}
\hline All & $3005 / 23013(13.1)$ & $2014 / 5993(33.6)$ & $<0.001$ \\
\hline$\leq 80$ & $2629 / 20840(12.6)$ & $1521 / 4776(31.8)$ & $<0.001$ \\
\hline$>80$ & $376 / 2173(17.3)$ & $493 / 1217(40.5)$ & $<0.001$
\end{tabular}

No (\%) with congestive heart failure

\begin{tabular}{lccc}
\hline All & $1932 / 22840(8.5)$ & $277 / 3167(8.7)$ & 0.59 \\
\hline$\leq 80$ & $1581 / 20697(7.6)$ & $185 / 2579(7.2)$ & 0.39 \\
\hline 180 & $351 / 2143(16.4)$ & $92 / 588(15.6)$ & 0.67 \\
\hline
\end{tabular}

No (\%) with hypertension

\begin{tabular}{lccc}
\hline All & $14331 / 22875(62.6)$ & $4170 / 5896(70.7)$ & $<0.001$ \\
\hline$\leq 80$ & $12687 / 20683(61.3)$ & $3273 / 4704(69.6)$ & $<0.001$ \\
\hline$>80$ & $1644 / 2192(75)$ & $897 / 1192(75.3)$ & 0.87 \\
\hline No (\%) with atrial fibrillation & & \\
\hline All & $5835 / 22753(25.6)$ & $1712 / 5896(29.0)$ & $<0.001$ \\
\hline$\leq 80$ & $4837 / 20613(23.5)$ & $1147 / 4704(24.4)$ & 0.19 \\
\hline$>80$ & $998 / 2140(46.6)$ & $565 / 1192(47.4)$ & 0.67
\end{tabular}

* Of patients with baseline National Institutes of Health stroke scale score in SITS datasheet, two (0.32\%) in age group $31-40$, five $(0.30 \%)$ in age group $41-50$, nine $(0.25 \%)$ in age group $51-60$, six patients $(0.1 \%)$ in age group $61-70$, eight $(0.09 \%)$ in age group $71-80$, and two $(0.1 \%)$ in age group $81-90$ were coded as having baseline score of 0 and treated with alteplase. They were assumed to have neurological deficit considered potentially disabling but not measured by restricted rules of the scale (such as distal limb weakness). These few cases will have no material impact on findings.

\section{Outcomes among patients aged $>80$}

Among the 3439 patients aged over 80 with a 90 day score on the modified Rankin scale and baseline data on stroke severity, treatment with thrombolysis was associated with a significantly more favourable distribution of scores at three months compared with controls (fig 2). The adjusted odds ratio was 1.4 (1.3 to 1.6); $\mathrm{P}<0.001 ; \mathrm{n}=3439$ (fig 3 . $\mathrm{P}$ for test of proportionality assumption was $<0.05$. The unadjusted odds ratio was 1.4 (1.2 to 1.6$),(\mathrm{P}<0.001 ; \mathrm{n}=3472)$. Dichotomised outcomes were significantly more favourable for thrombolysed patients than controls (2.1 (1.7 to 2.5) for favourable outcome (score 0-2 on modified Rankin scale); 1.9 (1.5 to 2.3) for excellent outcome (score $0-1$ ); and 0.89 (0.76 to1.04) for mortality).

Our sensitivity analysis, in which we adjusted for age, sex, history of either diabetes or previous stroke, previous use of antithrombotics, baseline National Institutes of Health stroke severity score, and hypertension, yielded Cochran-Mantel-Haenszel $\mathrm{P}=0.003$ and proportional odds of 1.5 (1.3 to 1.8$)$ in favour of thrombolysis.

The results indicate that 8.2 patients aged $>80$ need to be treated for one more patient to achieve a modified Rankin scale score of $0-2$.

Association of thrombolysis with outcome by age groups Distributions of scores on the modified Rankin scale at 90 days were significantly better among thrombolysed patients than controls within each 10 year age group from 40 to 90 and, except among the small samples of patients younger than 30 and older than 90 , point estimates for the adjusted odds ratios were consistent across all age groups (fig 3).

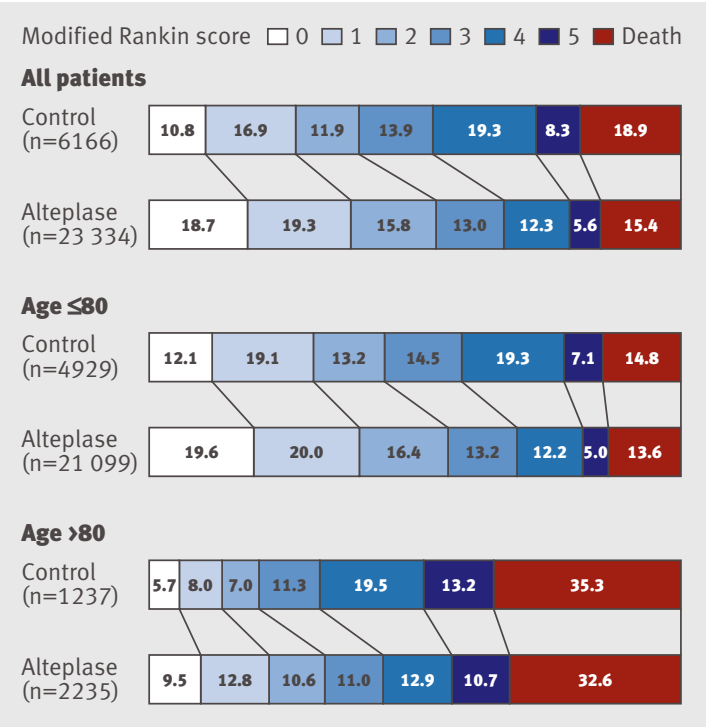

Fig 2 | Scores on modified Rankin scale (from $0=$ no symptoms from stroke to $6=$ death) at three months between patients who underwent thrombolysis with alteplase and controls, indicating shift towards improved outcomes with thrombolysis. Numbers within coloured cells are percentages thrombolysis.

The results indicate that 8.5 patients aged $\leq 80$ need to be treated for one more patient to achieve a modified Rankin scale score of $0-2$. 


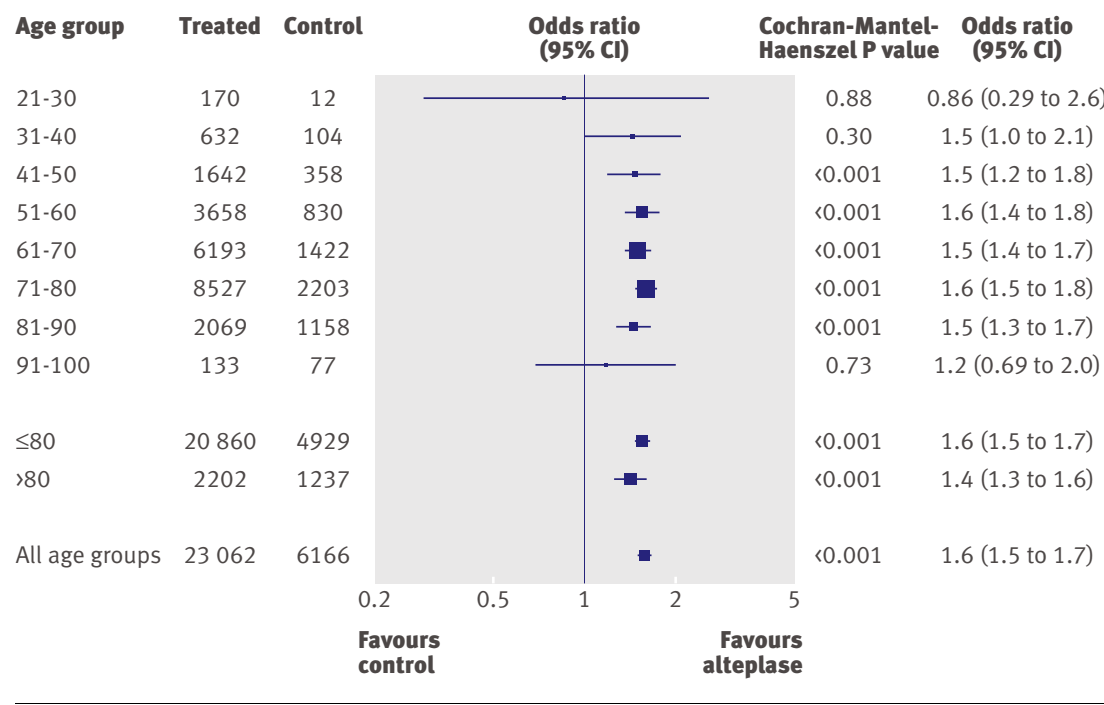

Fig 3 | Shift towards better outcomes on modified Rankin scale at three months adjusted for age and baseline severity (defined by National Institutes of Health stroke scale). Number of patients shown for age groups do not add up to 29228 because numbers of patients ages $<21$ $(n=38)$ and $>100(n=2)$ were too low to allow any comparison. All patients aged $<21$ were from SITS and underwent thrombolysis; 15 patients reached a 90 day modified Rankin score of 0 , 10 patients attained a score of 1 , eight patients reached a score of 2 , one patient achieved a score of 3, and two a score of 4; two died. Two patients aged 101 did not undergo thrombolysis in VISTA neuroprotection trials; they achieved modified Rankin score of 0 and 4 at 90 days difference). Data on onset to treatment time for use of alteplase were not collected in VISTA.

\section{DISCUSSION}

\section{Principal findings}

Comparing patients from SITS who were treated with alteplase at an average of 145 minutes after stroke onset against controls from VISTA who received no alteplase we found more favourable outcomes with alteplase across the entire range of scores on the modified Rankin scale (odds ratio 1.6, 95\% confidence interval 1.5 to $1.7, \mathrm{P}<0.001)$. The nature and extent of this effect of alteplase is comparable with results from pooled analysis of randomised controlled trials, confirming the validity of our controlled but non-randomised analysis. ${ }^{4}$ We were able to examine outcomes separately among patients aged $\leq 80$ and older patients aged $>80$. In each subgroup we found more favourable functional outcomes: odds ratios 1.6 (1.5 to 1.7$), \mathrm{n}=$ 25789 , and 1.4 (1.3 to 1.6 ), $\mathrm{n}=3439$, respectively.

Extending our analysis to smaller subgroups of age, we found independently significant benefits from alteplase in each 10 year age group from 40-90. We found no interaction between age and efficacy of alteplase and across the full age range from age under 20 to over 100. Only in patients aged under 30 did the trend not favour outcomes after use of alteplase.

In summary, we show that association between thrombolysis treatment and outcome is maintained in all patients, even in older patients, regardless of generally poorer outcomes in these age groups. with the effect of alteplase use on outcome in the entire dataset $(\mathrm{P}=0.45$ for score $0-2 v$ rest $)$, those aged $>80$ $(\mathrm{P}=0.76$ and 0.85 for score $0-2 v$ rest and $0-1 v$ rest, respectively), or those aged $\leq 80$ ( 0.42 and 0.48 for score $0-2 v$ rest and $0-1 v$ rest, respectively).

\section{Post-thrombolysis intracerebral haemorrhage}

The rate of symptomatic intracerebral haemorrhage per SITS-MOST definition ${ }^{27}(\geq 4$ point increase in National Institutes of Health stroke scale from baseline or death within 24 hours and parenchymatous haemorrhage (type $\mathrm{PH} 2$ or $\mathrm{PHr} 2$ ) at 22-36 hour imaging scans) was $2.5 \%(54 / 2163)$ among those aged $>80$ compared with $1.9 \%(398 / 20759)$ among those aged $\leq 80$, and thus not significantly higher (odds ratio 1.3, 0.96 to $1.8 ; \mathrm{P}=0.07)$. The corresponding rate for symptomatic intracerebral haemorrhage per National Institute of Neurological Disorders and Stroke definition ${ }^{27}$ (any increase in National Institutes of Health stroke scale from baseline and any parenchymal intracerebral haemorrhage) was significantly higher: $11.0 \%$ (229/2087) v 8.3\% (1670/20 220); 1.4, 1.2 to $1.6 ; \mathrm{P}<0.001)$.

\section{Onset to treatment time}

We calculated the time from onset of stroke to treatment for the administration of thrombolysis to patients in SITS-ISTR. The median time was similar in younger $(\leq 80)$ and older $(>80)$ patients $(145$ minutes, $\mathrm{P}=0.25$ for

\section{Strengths and limitations}

Our analysis of SITS-VISTA data is based on almost 30000 patients and confirms that there are improved outcomes after acute ischaemic stroke among patients who are offered thrombolytic therapy. The extent of the

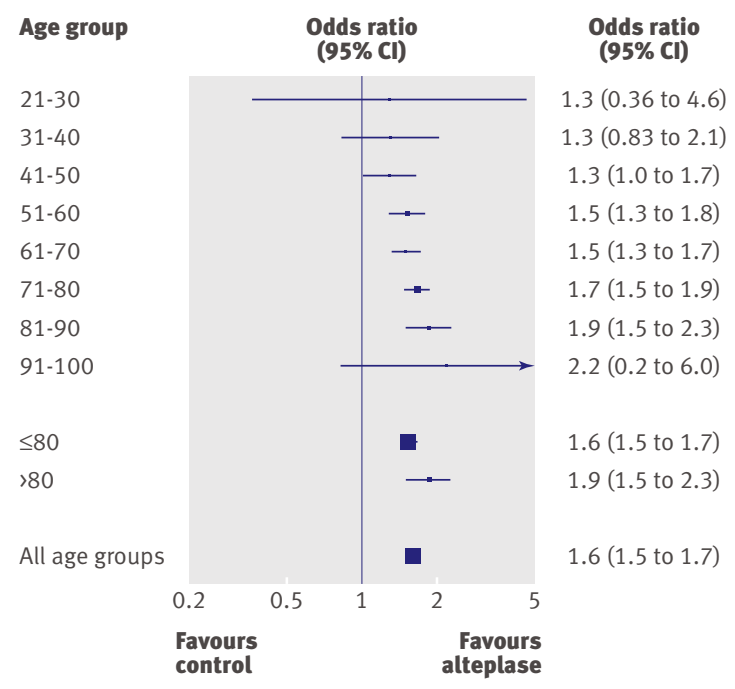

Fig 4 | Odds ratios for score 0-1 on modified Rankin scale at three months adjusted for age and baseline National Institutes of Health stroke severity scale in patients who received thrombolytic therapy 


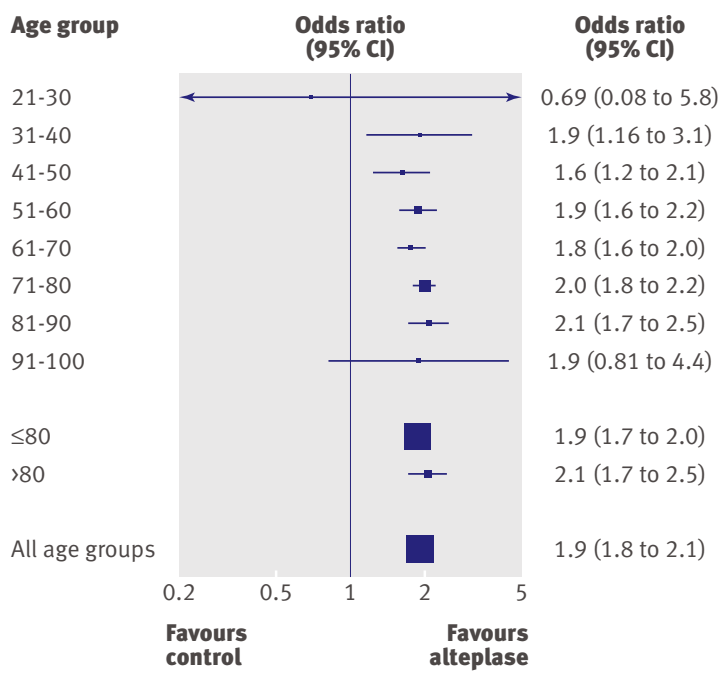

Fig 5 | Odds ratios for score $0-2$ on modified Rankin scale at three months adjusted for age and baseline National Institutes of Health stroke severity scale in patients who received thrombolytic therapy

apparent benefit matches that from published randomised trials. These observations extend to older age groups and only in a small group of patients aged 91100 (137 patients in alteplase group and 77 in the control group) did we fail to show significance, with consistent point estimates but wide confidence intervals. The point estimates for improved outcomes in this age group are also consistent with the published data. ${ }^{489}$

We undertook our primary analyses using "shift analysis," an analytical approach accepted by the European Drug Licensing agency. ${ }^{37}$ The CochranMantel-Haenszel test is a non-parametric approach that avoids invoking an assumption of a common odds ratio (that is, proportionality) across all cut points on the ordinal outcome scale. It provides a conservative estimate of significance. Because it does not express the extent of the association, we also applied an ordinal logistic regression analysis to estimate a common odds ratio across categories of the modified Rankin scale. Again, we found significantly better outcomes, though the proportionality assumption was not satisfied. Whereas a non-significant result for proportionality would imply that common odds could be assumed, the converse does not necessarily apply. ${ }^{44}$ The proportionality assumption test might be oversensitive when applied to large sample sizes. ${ }^{45}$ Furthermore, it is a global test that cannot differentiate the heterogeneity resulting from alteplase or other covariates $^{45}$ As our sample sizes were large we could still be justified in using the odds estimated from ordinal logistic regression. Even so, for final confirmation we used a less powerful dichotomised approach. ${ }^{3845}$ With all three methods we reach similar conclusions. ${ }^{385051}$

There were improved outcomes among patients who underwent thrombolysis in age groups from 31 to 90 . We found no improved outcomes for patients aged under 30 and above 90 , but the small number of patients in these groups greatly reduced statistical power for these analyses and the trends mostly followed the same pattern as for intermediate ages.

We chose age and baseline National Institutes of Health stroke scale score for adjusted analysis mainly because of their established roles of influence on stroke outcomes..$^{5253}$ We also undertook sensitivity analysis, adjusting for differences in age, sex, history of diabetes or previous stroke, previous use of an antithrombotic, baseline National Institutes of Health stroke scale score, and hypertension, between those who did and did not undergo thrombolysis. ${ }^{34}$ The adjusted analyses for these variables confirmed significant findings for improved outcomes with thrombolysis regardless of age.

The baseline demographic characteristics for the complete dataset favoured the thrombolysis group. This influence, however did not extend to patients aged $>80$. As a result, though our estimates of overall effect of alteplase could be biased, the relative differences between subgroups should remain reliable. We did not match patients by comorbidity score or baseline functional status, which could be considered a limitation. Premorbid functional status, however, is difficult to establish reliably in patients with stroke, and the strong influence of baseline severity on outcome, for which we did adjust, is known. ${ }^{54} \mathrm{~A}$ corresponding analysis examining the influence of baseline severity on outcomes in SITS and VISTA data is under way. ${ }^{55}$

Our conclusions derive merit from being based on a huge population of patients who were treated in routine clinical practice $(\mathrm{n}=28136)$ and compared against controls from rigorously conducted neuroprotection trials: any bias in quality of care should favour the control group. The limitation of SITS-ISTR data has been discussed extensively in previous publications. ${ }^{5272856} \mathrm{In}$ short, SITS-ISTR is a registry, and it is therefore impossible to guarantee completeness of inclusions and to exclude selection bias. ${ }^{27}$ For a sample of patients included in SITS-MOST (monitoring study) ( $\mathrm{n}=6483)$, source data were verified onsite by monitors under the

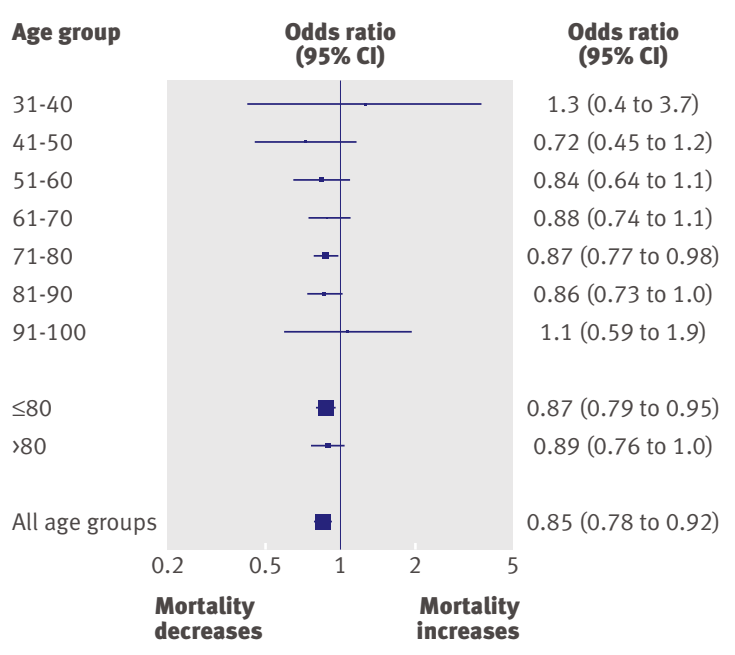

Fig 6 | Odds ratios for mortality at three months adjusted for age and baseline National Institutes of Health stroke severity scale in patients who received thrombolytic therapy 


\section{WHAT IS ALREADY KNOWN ABOUT THIS TOPIC}

In the European Union patients with ischaemic stroke aged $>80$ are not recommended for thrombolysis, though no one has shown negative outcomes

Age is a non-modifiable risk factor for stroke, and the proportion of older patients is rapidly rising in our society

\section{WHAT THIS STUDY ADDS}

Odds for improved outcomes in patients with ischaemic stroke who receive thrombolysis (with alteplase) are similar in those aged $\leq 80$ and $>80$

Age alone should not be a barrier to thrombolysis

supervision of the relevant national coordinator. ${ }^{27}$ The monitors could examine admissions department records, etc, to check for completeness of data. ${ }^{27}$ Investigators agreed to register their patients soon after admission, before outcome was known. ${ }^{27}$ Individual investigators' results are not published, limiting the incentive for selection; in contrast, the sharing of total enrolment numbers might act as an incentive to be inclusive $^{27}$ The almost identical main outcomes in SITS-MOST ${ }^{27}$ and randomised controlled trials ${ }^{56}$ after adjustment for baseline differences suggests that the influence from such potential bias is limited. Subsequent studies based on SITS-ISTR data also show the similar outcome for the overall study population compared with the SITS-MOST. ${ }^{5}$ Selective reporting of good outcomes in SITS could generate an opposite bias but was limited through site monitoring procedures used in the SITS-MOST study. ${ }^{25}$ None of the neuroprotective agents used for the patients in the VISTA control group has an influence on outcome, and over half of the VISTA cohort received only placebo.

Because VISTA lacks data on repeat brain imaging among patients who did not receive thrombolysis, we had no data on symptomatic intracerebral haemorrhage in our control group. Therefore, we compared the rates between patients aged $>80$ and $\leq 80$ only with SITS data. There was no difference in rates between those aged $>80$ and $\leq 80$ with the SITS-MOST definition but slightly higher with the NINDS definition. In a complementary per protocol analysis of SITS-ISTR data (that is, patients' selection based on SITS-MOST criteria $^{27}$ except for its age criterion), there was no significant difference in rates of symptomatic intracerebral haemorrhage among patients aged $>80$ compared with the younger cohort $(1.8 \%$ v $1.7 \%$, $\mathrm{P}=0.70$, adjusted odds ratio $0.90,0.73$ to 1.09$).{ }^{57}$ Our SITS patients in the SITS-VISTA dataset are unselected and therefore rates of symptomatic intracerebral haemorrhage are slightly higher in the current study. Regardless, we have shown that even if there were any more haemorrhages among elderly patients who receive thrombolysis, based on a conservative definition, there seems to be no adverse influence on the distribution of outcomes. In fact, we observe a beneficial effect on mortality. Others have concluded that factors such as comorbidity, rather than use of alteplase, are responsible for the observed increase in late case fatality among older patients. ${ }^{2158}$

\section{Comparison with other studies}

We reached the same conclusions as analyses that used VISTA data or the limited pooled randomised trial data in elderly patients. ${ }^{59}$ Elderly patients treated with thrombolysis in trials reported by VISTA $(n=5817)$ had significantly better adjusted outcomes than patients who did not receive thrombolysis (odds ratio $1.3,1.1$ to $1.7 ; \mathrm{P}=0.002) .{ }^{59}$ Elderly patients treated in the pooled randomised trials showed a trend towards better adjusted outcomes (score on modified Rankin scale 0-2 $v 3-6$ ) than those who did nor receive thrombolysis $(1.8,0.73$ to $4.3 ; \mathrm{n}=137) .{ }^{59}$ Previous studies have shown findings consistent with our results but on small datasets. ${ }^{205860-64}$

Despite these points, treatment allocation in our study was not randomised. More extensive data from randomised controlled trials could more conclusively answer this question. Two trials are currently examining this topic. ${ }^{6566} \mathrm{~A}$ trial in Italy has so far enrolled around $17 \%$ of the planned 600 patients over a two year period. ${ }^{65}$ In the UK, the International Stroke Trial-3 (IST-3) is examining outcomes among all patients who receive thrombolysis and has no upper age limit in its inclusion criteria. ${ }^{66}$ Over 12 years, the trial has enrolled around 2400 patients of the originally planned 6000. About a third of these patients are aged $>80$ and are being treated within the time window of interest. ${ }^{667}$

\section{Conclusions and policy implication}

In our analysis, patients who were treated with intravenous alteplase had better outcomes than untreated patients, and this effect was not dependent on age. In particular, patients aged over 80 derived similar benefit from treatment as younger patients. The weight of evidence to date indicates a potential for benefit in the older people, and there is no a priori reason to suspect a diminished effect compared with younger people. Furthermore there are reassuring safety data on the risk of intracerebral haemorrhage. We conclude that clinical treatment guidelines should be revised to remove the age restriction in use of intravenous alteplase for acute ischaemic stroke. Age alone should not be a barrier to treatment.

The SITS steering committee members were A Davalos, M Grond, G Ford, W Hacke, M Hennerici, M Kaste, V Larrue, KR Lees, RO Roine, D Toni, and $\mathrm{N}$ Wahlgren. The VISTA steering committee members were

A Alexandrov, PW Bath, E Bluhmki, L Claesson, J Curram, SM Davis, G Donnan, HC Diener, M Fisher, B Gregson, J Grotta, W Hacke, MG Hennerici, M Hommel, M Kaste, KR Lees (chair), P Lyden, J Marler, K Muir, R Sacco, A Shuaib, P Teal, NG Wahlgren, S Warach, and C Weimar. The data were presented by NKM at the European Stroke Conference, 2010. The analyses were based on a research proposal approved both by the VISTA steering committee and the SITS-ISTR.

Contributors: NKM, NGW, and NA had access to the SITS-ISTR data. NKM and KRL had access to the VISTA data. NKM and KRL designed and interpreted the analyses and drafted the manuscript. NGW, NA, GA, JEH, PJL, and PAR contributed SITS-ISTR data, reviewed the outline proposal, commented on the manuscript, and approved the final version. All 
authors take full responsibility for the content. The manuscript was reviewed and approved by the steering committees of VISTA and SITS. No commercial organisation was involved in the origination, execution or reporting of this work. NKM is guarantor.

Funding: VISTA has received financial support from the European Stroke Organisation in the form of an unrestricted grant and contributions towards data extraction and capacity building from the Universities of Glasgow, California San Diego, Nottingham, Edmonton, Calgary, Texas, and Massachusetts; from commercial groups including Brainsgate, Novartis, Boehringer Ingelheim, and the Vertical Group; and from grant agencies and charities including the UK Stroke Association. SITS-ISTR is funded by an unrestricted grant from Boehringer Ingelheim, Ferrer, and grant from European Union Public Health Executive Authority (PHEA) Financial support was also provided through the regional agreement on medical training and research (ALF) between Stockholm County Council and the Karolinska Institute. NKM is supported by a Scottish Overseas Research Studentship, a University of Glasgow scholarship, and an educational grant from the European Stroke Organisation for Young Neurologists. PIL is supported by the Finnish Academy and Sigrid Juselius foundation and Helsinki University Hospital research funds (EVO). Competing interests: All authors have completed the Unified Competing Interest form at www.icmje.org/coi_disclosure.pdf (available on request from the corresponding author) and declare: KRL has received honorariums from Boehringer Ingelheim, Lundbeck, Thrombogenics, Talecris. GA is a member of the advisory board for Boehringer Ingelheim Denmark. JAE was investigator of ECASS II, ECASS III, and PROFESS promoted by Boehringer Ingelheim. NA is an employee of SI TS International, which received a grant from Boehringer Ingelheim for the SITS-MOST/SITS-ISTR study with alteplase. NGW has received expenses from Boehringer Ingelheim for his role as member of the steering committee in relation to the ECASS III trial with alteplase and served as a consultant to Thrombogenics as chairman of the DSMB. SITS International (chaired by NGW) received a grant from Boehringer Ingelheim and from Ferrer for the SITS-MOST/SITS-ISTR. His institution has also received grant support towards administrative expenses for coordination of the ECASS III trial. NGW has also received lecture fees from Boehringer Ingelheim and from Ferrer. PAR is the German deputy national SITS coordinator. He was investigator of ECASS III and PROFESS sponsored by Boehringer Ingelheim and of DIAS and DIAS-2 sponsored by PAION. He received honorariums and travel expenses from Boehringe Ingelheim, PAION, and Ferrer.

Ethical approval: Not required.

Data sharing: Data sharing: no additional data available.

1 Generalized efficacy of t-PA for acute stroke. Subgroup analysis of the NINDS t-PA Stroke Trial. Stroke 1997;28:2119-25.

2 National Institute of Neurological Disorders and Stroke rt-PA Stroke Study Group. Tissue plasminogen activator for acute ischemic stroke. N Engl J Med 1995;333:1581-7.

3 Hacke W, Kaste M, Bluhmki E, Brozman M, Davalos A, Guidetti D, et al. Thrombolysis with alteplase 3 to 4.5 hours after acute ischemic stroke. N Engl J Med 2008;359:1317-29.

4 Lees KR, Bluhmki E, von Kummer R, Brott TG, Toni D, Grotta JC, et al. Time to treatment with intravenous alteplase and outcome in stroke: an updated pooled analysis of ECASS, ATLANTIS, NINDS, and EPITHET trials. Lancet 2010;375:1695-703.

5 Ahmed N, Wahlgren N, Grond M, Hennerici M, Lees KR, Mikulik R, et al. Implementation and outcome of thrombolysis with alteplase 3 $4.5 \mathrm{~h}$ after an acute stroke: an updated analysis from SITS-ISTR. Lancet Neurol 2010;9:866-74.

6 Bonita R, Anderson CS, Broad JB, Jamrozik KD, Stewart-Wynne EG Anderson NE. Stroke incidence and case fatality in Australasia. A comparison of the Auckland and Perth population-based stroke registers. Stroke 1994;25:552-7.

7 Marini C, Baldassarre M, Russo T, De Santis F, Sacco S, Ciancarelli I, et al. Burden of first-ever ischemic stroke in the oldest old: evidence from a population-based study. Neurology 2004;62:77-81.

8 Di Carlo A, Baldereschi M, Gandolfo C, Candelise L, Ghetti A, Maggi S, et al. Stroke in an elderly population: incidence and impact on survival and daily function. Italian Longitudinal Study on Aging. Cerebrovasc Dis 2003;16:141-50.

9 Derex L, Nighoghossian N. Thrombolysis, stroke-unit admission and early rehabilitation in elderly patients. Nat Rev Neurol 2009;5:506-11.

10 Hacke W, Kaste M, Fieschi C, Toni D, Lesaffre E, von Kummer R, et al. Intravenous thrombolysis with recombinant tissue plasminogen activator for acute hemispheric stroke. European Cooperative Acute Stroke Study (ECASS). JAMA 1995;274:1017-25.
11 Hacke W, Albers G, Al-Rawi Y, Bogousslavsky J, Davalos A, Eliasziw M, et al. The Desmoteplase in Acute Ischemic Stroke Trial (DIAS): a phase II MRI-based 9-hour window acute stroke thrombolysis trial with intravenous desmoteplase. Stroke 2005;36:66-73

12 Hacke W, Furlan AJ, Al-Rawi Y, Davalos A, Fiebach JB, Gruber F, et al. Intravenous desmoteplase in patients with acute ischaemic stroke selected by MRI perfusion-diffusion weighted imaging or perfusion CT (DIAS-2): a prospective, randomised, double-blind, placebocontrolled study. Lancet Neurol 2009;8:141-50.

13 Wahlgren N. Systemic thrombolysis in clinical practice: what have we learned after the Safe Implementation of Thrombolysis in Stroke Monitoring Study? Cerebrovasc Dis 2009;27(suppl 1):168-76.

14 Lees KR, Ford GA, Muir KW, Ahmed N, Dyker AG, Atula S, et al. Thrombolytic therapy for acute stroke in the United Kingdom: experience from the safe implementation of thrombolysis in stroke (SITS) register. Q J Med 2008;101:863-9.

15 Ringleb PA, Schwark C, Kohrmann M, Kulkens S, Juttler E, Hacke W, et al. Thrombolytic therapy for acute ischaemic stroke in octogenarians: selection by magnetic resonance imaging improves safety but does not improve outcome. J Neurol Neurosurg Psychiatry 2007;78:690-3.

16 Committee for the Proprietary Medicinal Products. Summary information on a referral opinion following an arbitration persuant to article 29 of directive 2001/83/EC, for actilyse. EMEA, 2002 (No: CPMP/3346/02-EN). www.ema.europa.eu/pdfs/human/referral/ Actilyse/334602en.pdf.

17 Hacke W, Donnan G, Fieschi C, Kaste M, von Kummer R, Broderick JP, et al. Association of outcome with early stroke treatment: pooled analysis of ATLANTIS, ECASS, and NINDS rt-PA stroke trials. Lancet 2004;363:768-74.

18 Heuschmann PU, Kolominsky-Rabas PL, Roether J, Misselwitz B, Lowitzsch K, Heidrich J, et al. Predictors of in-hospital mortality in patients with acute ischemic stroke treated with thrombolytic therapy. JAMA 2004;292:1831-8.

19 Bateman BT, Schumacher HC, Boden-Albala B, Berman MF, Mohr JP, Sacco RL, et al. Factors associated with in-hospital mortality after administration of thrombolysis in acute ischemic stroke patients: an analysis of the nationwide inpatient sample 1999 to 2002. Stroke 2006;37:440-6.

20 Engelter ST, Bonati LH, Lyrer PA. Intravenous thrombolysis in stroke patients of $>$ or $=80$ versus $<80$ years of age-a systematic review across cohort studies. Age Ageing 2006;35:572-80.

21 Fonarow GC, Reeves MJ, Zhao X, Olson DM, Smith EE, Saver JL, et al. Age-related differences in characteristics, performance measures, treatment trends, and outcomes in patients with ischemic stroke. Circulation 2010;121:879-91.

22 Foerch C, Sitzer M, Steinmetz H, Neumann-Haefelin T. Future demographic trends decrease the proportion of ischemic stroke patients receiving thrombolytic therapy: a call to set-up therapeutic studies in the very old. Stroke 2009;40:1900-2.

23 Dunnell K. Ageing and mortality in the UK-national statistician's annual article on the population. Popul Trends 2008;134:6-23.

24 Europeans are living longer. Official website of European Union. http://europa.eu/abc/keyfigures/sizeandpopulation/older/ index_en.htm

25 Ali M, Atula S, Bath PM, Grotta J, Hacke W, Lyden P, et al. Stroke outcome in clinical trial patients deriving from different countries. Stroke 2009;40:35-40.

26 Ali M, Bath PM, Curram J, Davis SM, Diener HC, Donnan GA, et al. The virtual international stroke trials archive. Stroke 2007;38:1905-10.

27 Wahlgren N, Ahmed N, Davalos A, Ford GA, Grond M, Hacke W, et al. Thrombolysis with alteplase for acute ischaemic stroke in the Safe Implementation of Thrombolysis in Stroke-Monitoring Study (SITSMOST): an observational study. Lancet 2007;369:275-82.

28 Wahlgren N, Ahmed N, Davalos A, Hacke W, Millan M, Muir K, et al. Thrombolysis with alteplase 3-4.5 h after acute ischaemic stroke (SITS-ISTR): an observational study. Lancet 2008;372:1303-9.

29 Chacon MR, Jensen MB, Sattin IA, Zivin IA Neuroprotection in cerebral ischemia: emphasis on the SAINT trial. Curr Cardiol Rep 2008;10:37-42

30 Quinn TJ, Dawson J, Walters M. Dr John Rankin; his life, legacy and the 50th anniversary of the Rankin stroke scale. Scott Med 2008;53:44-7.

31 Internet Stroke Center at Washington University in St Louis. www. strokecenter.org/.

32 Lees KR, Zivin JA, Ashwood T, Davalos A, Davis SM, Diener HC, et al. NXY-059 for acute ischemic stroke. N Engl / Med 2006:354:588-600.

33 Lees KR, Davalos A, Davis SM, Diener HC, Grotta J, Lyden P, et al. Additional outcomes and subgroup analyses of NXY-059 for acute ischemic stroke in the SAINT I trial. Stroke 2006;37:2970-8.

34 Gray LI, Bath PM, Collier T. Should stroke trials adjust functional outcome for baseline prognostic factors? Stroke 2009;40:888-94. 
35 Saver JL, Yafeh B. Confirmation of tPA treatment effect by baseline severity-adjusted end point reanalysis of the NINDS-tPA stroke trials. Stroke 2007;38:414-6.

36 Mann J. tPA for acute stroke: balancing baseline imbalances. CMAJ 2002;166:1651-2; author reply 1652-3.

37 Committee for Proprietary Medicinal Products, London. Points to consider on clinical investigation of medicinal products for the treatment of acute stroke. European Agency for the Evaluation of Medicinal Products, Sep 2001 (No: CPMP/EWP/560/98). www.tga. gov.au/docs/pdf/euguide/ewp/056098en.pdf.

38 Saver JL, Gornbein J. Treatment effects for which shift or binary analyses are advantageous in acute stroke trials. Neurology 2009;72:1310-5.

39 Fedorov V, Mannino F, Zhang R. Consequences of dichotomization. Pharm Stat 2009;8:50-61.

40 Senn S, Julious S. Measurement in clinical trials: a neglected issue for statisticians? Stat Med 2009;28:3189-209.

41 Bluhmki E, Windeler ], Hacke W. [Neurological scales as endpoints in stroke studies. Aspects of statistical evaluation]. Nervenarzt 2000;71:797-801.

42 Savitz SI, Lew R, Bluhmki E, Hacke W, Fisher M. Shift analysis versus dichotomization of the modified Rankin scale outcome scores in the NINDS and ECASS-II trials. Stroke 2007;38:3205-12.

43 Saver JL. Novel end point analytic techniques and interpreting shifts across the entire range of outcome scales in acute stroke trials. Stroke 2007;38:3055-62.

44 Gameroff MJ. Using the proportional odds model for health-related outcomes: Why, when, and how with various SAS procedures. Statistics and data analysis. SUGI 30 Proceedings. Paper 205-30. www2.sas.com/proceedings/sugi30/toc.html\#st.

45 Scott SC, Goldberg MS, Mayo NE. Statistical assessment of ordinal outcomes in comparative studies. J Clin Epidemiol 1997;50:45-55.

46 Engelter ST, Reichhart M, Sekoranja L, Georgiadis D, Baumann A, Weder B, et al. Thrombolysis in stroke patients aged 80 years and older: Swiss survey of IV thrombolysis. Neurology 2005;65:1795-8.

47 Von Elm E, Altman DG, Egger M, Pocock SJ, Gotzsche PC Vandenbroucke JP. Strengthening the Reporting of Observational Studies in Epidemiology (STROBE) statement: guidelines for reporting observational studies. BMJ 2007;335:806-8.

48 Mateen F), Buchan AM, Hill MD, CASES Investigators. Outcomes of thrombolysis for acute ischemic stroke in octogenarians versus nonagenarians. Stroke 2010;41:1833-5.

49 Lees KR, Bluhmki E, Toni D, von Kummer R. MJ, Brott TG, et al. Time dependent response to treatment with intravenous rtPA for stroke: an updated pooled analysis of ECASS, ATLANTIS and NINDS stroke trials. Stroke 2010;41:e200.

50 Bath PM, Gray LJ, Collier T, Pocock S, Carpenter J. Can we improve the statistical analysis of stroke trials? Statistical reanalysis of functional outcomes in stroke trials. Stroke 2007;38:1911-5.

51 Brown DL, Coffey CS. Stroke trials: a shift to shift analysis? Neurology 2009;72:1292-3.

52 Weimar C, Konig IR, Kraywinkel K, Ziegler A, Diener HC. Age and National Institutes of Health stroke scale score within 6 hours after onset are accurate predictors of outcome after cerebral ischemia: development and external validation of prognostic models. Stroke 2004;35:158-62.

53 Konig IR, Ziegler A, Bluhmki E, Hacke W, Bath PM, Sacco RL, et al. Predicting long-term outcome after acute ischemic stroke: a simple index works in patients from controlled clinical trials. Stroke 2008;39:1821-6.
54 Mishra NK, Lyden P, Grotta JC, Lees KR, for the VISTA Collaborators. Thrombolysis is associated with consistent functional improvement across baseline stroke severity. A comparison of outcomes in patients from the virtual international stroke trials archive (VISTA). Stroke 2010;41:2612-7.

55 Ahmed A, Mishra NK, Kobayashi A, Käll T-B, Ringleb P, Lees KR, et al. Improved outcomes after thrombolysis across baseline NIHSS scores 5-24: controlled comparison of the SITS international registry (SITS-ISTR) versus Virtual International Stroke Trials Archive (VISTA). European Stroke Conference, 2010. Cerebrovasc Dis 2010;29(suppl 2):1-341.www.esc-archive.eu/barcelona10/ bar author 1.asp.

56 Wahlgren N, Ahmed N, Eriksson N, Aichner F, Bluhmki E, Davalos A et al. Multivariable analysis of outcome predictors and adjustment of main outcome results to baseline data profile in randomized controlled trials: Safe Implementation of Thrombolysis in StrokeMOnitoring STudy (SITS-MOST). Stroke 2008;39:3316-22.

57 Ford GA Ahmed N, Azevedo E, Grond M, Larrue V, Lindsberg P, et al. Intravenous alteplase for stroke in those older than 80 years old. Stroke 2010;41:2568-74.

58 Uyttenboogaart M, Schrijvers EM, Vroomen PC, De Keyser J, Luijckx GJ. Routine thrombolysis with intravenous tissue plasminogen activator in acute ischaemic stroke patients aged 80 years or older: a single centre experience. Age Ageing 2007;36:577-9.

59 Mishra NK, Diener H-C, Lyden PD, Bluhmki E,Lees KR, for the VIS TA Collaborators. Influence of age on outcome from thrombolysis in acute stroke: a controlled comparison in patients from the virtual international stroke trials archive (VISTA). Stroke 2010; Oct 28, epub ahead of print.

60 Poppe AY, Hill MD. Acute ischaemic stroke in patients aged 80 years and older: focus on the tolerability of thrombolytic agents. Drugs Aging 2008;25:95-103.

61 Vatankhah B, Dittmar MS, Fehm NP, Erban P, Ickenstein GW, Jakob W, et al. Thrombolysis for stroke in the elderly. I Thromb Thrombolysis 2005;20:5-10.

62 Sylaja PN, Cote R, Buchan AM, Hill MD. Thrombolysis in patients older than 80 years with acute ischaemic stroke: Canadian Alteplase for Stroke Effectiveness Study. J Neurol Neurosurg Psychiatry 2006;77:826-9.

63 Toni D, Lorenzano S, Agnelli G, Guidetti D, Orlandi G, Semplicini A, et al. Intravenous thrombolysis with rt-PA in acute ischemic stroke patients aged older than 80 years in Italy. Cerebrovasc Dis 2008;25:129-35.

64 Mateen FJ, Buchan AM, Hill MD. Outcomes of thrombolysis for acute ischemic stroke in octogenarians versus nonagenarians. Stroke 2010;41:1833-5.

65 Toni D, Lorenzano S. Intravenous thrombolysis with rt-PA in acute stroke patients aged >or=80 years. Int J Stroke 2009;4:21-2.

66 Sandercock P, Lindley R, Wardlaw I, Dennis M, Lewis S, Venables G, et al. The third international stroke trial (IST-3) of thrombolysis for acute ischaemic stroke. Trials 2008;9:37.

67 Sandercock P. Thrombolysis of patients >80: a different view. UK Stroke Forum, 2009. www.dcn.ed.ac.uk/ist3/ Glasgow_UKCRN_Dec2009/

Thrombolysis for over 80 UKSF debate dec 2009 sandercock. ppt.

Accepted: 7 September 2010 